\title{
Rolling at small scales
}

Nielsen, Kim L.; Niordson, Christian F.; Hutchinson, John W.

\section{Published in:}

Journal of Manufacturing Science and Engineering

Link to article, DOI:

$10.1115 / 1.4031068$

Publication date:

2016

Document Version

Peer reviewed version

Link back to DTU Orbit

Citation (APA):

Nielsen, K. L., Niordson, C. F., \& Hutchinson, J. W. (2016). Rolling at small scales. Journal of Manufacturing Science and Engineering, 138(4), [041004]. https://doi.org/10.1115/1.4031068

\section{General rights}

Copyright and moral rights for the publications made accessible in the public portal are retained by the authors and/or other copyright owners and it is a condition of accessing publications that users recognise and abide by the legal requirements associated with these rights.

- Users may download and print one copy of any publication from the public portal for the purpose of private study or research.

- You may not further distribute the material or use it for any profit-making activity or commercial gain

- You may freely distribute the URL identifying the publication in the public portal

If you believe that this document breaches copyright please contact us providing details, and we will remove access to the work immediately and investigate your claim 


\title{
Rolling at Small Scales
}

\author{
Kim L. Nielsen* \\ Associate Professor \\ Department of Mechanical Engineering \\ Technical University of Denmark \\ DK-2800 Kongens Lyngby \\ Email: kin@mek.dtu.dk
}

\author{
Christian F. Niordson \\ Associate Professor \\ Department of Mechanical Engineering \\ Technical University of Denmark \\ DK-2800 Kongens Lyngby \\ Email: cn@mek.dtu.dk
}

\author{
John W. Hutchinson \\ Professor \\ School of Engineering and Applied Sciences \\ Harvard University \\ Cambridge, MA 02138, USA \\ Email: jhutchin@fas.harvard.edu
}

The rolling process is widely used in the metal forming industry, and has been so for many years. However, the process has attracted renewed interest as it recently has been adapted to very small scales where conventional plasticity theory cannot accurately predict the material response. It is well-established that gradient effects play a role at the micron scale, and the objective of this study is to demonstrate how strain gradient hardening affects the rolling process. Specifically, the paper addresses how the applied roll torque, roll forces, and the contact conditions are modified by strain gradient plasticity. Metals are known to be stronger when large strain gradients appear over a few microns; hence the forces involved in the rolling process are expected to increase relatively at these smaller scales. In the present numerical analysis, a steady-state modeling technique that enables convergence without dealing with the transient response period is employed. This allows for a comprehensive parameter study. Coulomb friction, including a stick-slip condition, is used as a first approximation. It is found that length scale effects increase both the forces applied to the roll, the roll torque, and thus the power input to the process. The contact traction is also affected, particularly for sheet thicknesses on the order of $10 \mu m$ and below. The influences of the length parameter and the friction coefficient are emphasized, and results are presented for multiple sheet reductions and roll sizes.

Keywords: Size effects, Metal forming, Rolling, Foils

\section{Nomenclature \\ $\dot{a} \quad$ Sheet velocity.}

*Address all correspondence to this author (Tel: +45 4525-4258).
$A^{(e)} \quad$ Element area.

$b$ Sheet width in out-of-plane direction.

$E$ Young's modulus.

$E^{p}, \dot{E}^{p} \quad$ Enriched effective plastic strain and strain rate.

$F_{\text {Pull }} \quad$ Full force along rolling direction.

$F_{\text {Punch }} \quad$ Punch force perpendicular to sheet.

$F_{n}^{(e)}, F_{f}^{(e)} \quad$ Normal force and friction force.

$h, H$ Deformed and undeformed sheet thickness.

$L^{(e)}$ Element length.

$L_{D} \quad$ Dissipative length parameter.

$m$ Strain rate hardening exponent.

$M_{i j} \quad$ Higher order tractions.

$N^{(n)}$ Shape function belonging to node $n$.

$N$ Strain hardening exponent.

$P$ Power input.

$q_{i j}, q_{i j}^{D} \quad$ Total micro-stress, and dissipative micro-stress.

$R$ Roll radius.

$t$ Time.

$T$ Roll torque.

$T_{i} \quad$ Nominal surface traction.

$u_{i}$ Displacements.

$x_{i} \quad$ Cartesian coordinates.

$\Delta$ Punch displacement.

$\dot{\varepsilon}_{0} \quad$ Reference strain rate.

$\varepsilon_{i j, k}^{p}, \dot{\varepsilon}_{i j, k}^{p} \quad$ Plastic strain gradient and strain gradient rates.

$\varepsilon_{i j}, \varepsilon_{i j}^{e}, \varepsilon_{i j}^{p} \quad$ Total strain, elastic strain, and plastic strain.

$\mathcal{L}_{i j k l}$ Elastic moduli.

$\mu$ Friction coefficient.

$v$ Poison ratio.

$\sigma_{C} \quad$ Gradient enriched effective stress.

$\sigma_{i j}, s_{i j} \quad$ Cauchy stress and stress diviator.

$\sigma_{Y} \quad$ Initial yield stress. 
$\tau_{i j k}, \tau_{i j k}^{D} \quad$ Total higher order stress and dissipative higher order stress.

$\Psi \quad$ Free energy.

\section{Introduction}

The flat rolling process is widespread in the metal forming industry, and it is applied to sheet thicknesses ranging from several centimeters to fractions of millimeters (foil rolling). The process has found multiple uses and it is under constant development for new applications and new materials (see e.g. [1] for an overview). Currently, there is a push towards down scaling the process aiming for highthroughput of ever thinner foils. Efforts are also underway to create very small scale features by continuous imprinting with the rolling process (so-called roll-molding, [2]). Compared to flat rolling, the deformation field developed during roll-molding is more complex as three dimensional features are allowed to form. However, even during flat rolling, a non-homogeneous deformation of the sheet takes place as it is being forced between the rolls, making the contact conditions and elastic unloading essential features of the model solution [3-6]. Thus, strain gradients must be expected to evolve.

With large strain gradients come increased hardening at the micron scale. The explanation for this is now generally accepted to lie in the concept of Geometrically Necessary Dislocations (GND's). GND's must necessarily be stored when large plastic strain gradients appear [7], and this gives rise to free energy associated with the local stress field of the GND's, as-well as increased dissipation when the GND's move in the lattice. At small scales, GND's can become a substantial portion of the total dislocation density, which is normally dominated by so-called Statistically Stored Dislocations (SSD's) at larger scales. Thus, a relatively larger amount of energy is required to deform the material at small scales in the presence of gradients, and this leads to an apparent increase in yield stress and strain hardening. With rolling at a small scale in mind, the questions to be addressed are; when do the size effects influence the rolling process? and; how will strain gradient hardening affect the applied roll torquelforce and the contact conditions?

To tackle these issues, and to develop an accurate numerical framework, the material model must represent stresses over the full range of length scales involved. For this, a vast amount of theoretical literature seeking to encapsulate the experimentally observed gradient effects at micron scale has been put forward [8-20]. The higher order elasticviscoplastic theory by Fleck and Willis [15] is employed in the current study, and the concept of higher order stresses, work conjugate to the strain gradients, is adopted to widen the range of length scales for which the model is valid. Moreover, focus is on the steady-state rolling solution. Thus, transient effects initiating the rolling process will not be considered in the present study. For an efficient modeling approach, the steady-state finite element formulation proposed by Dean and Hutchinson [21], suitable to history dependent material deformation, will be adapted to the rolling in the present study (see also [6] for a similar approach in a conventional plasticity context).

The paper is structured as follows. The material model and steady-state formulation are presented in Section 2, while the boundary value problem is outlined in Section 3. A steady-state modeling framework that enables convergence, without having to deal with the transient response of the rolling process, is employed. The results are laid out in Section 4 , and a conclusion is given in Section 5.

\section{Model: Constitutive Relations, Steady-State Formu- lation, and Contact Procedure}

\subsection{Rate-Sensitive Constitutive Material Model}

The flat rolling problem is analyzed using the gradient enhanced elastic-viscoplastic material model proposed in $[11,12,15]$. Here, a small strain formulation is employed. This is a reasonable approximation to the rolling process as the overall straining is proportional to the sheet reduction when limiting this to $\sim 15 \%$. For small sheet reductions, the strains and the rotations remain small - yet large plastic strain gradients can evolve (see e.g. Fig. 7). An additive decomposition of the total strain is applied, so that $\varepsilon_{i j}=\varepsilon_{i j}^{e}+\varepsilon_{i j}^{p}$, where $\varepsilon_{i j}^{e}$ is the elastic part and $\varepsilon_{i j}^{p}$ is the plastic part. The total strain field is determined from the displacements, which together with the plastic strain components are determined based on the principle of virtual work for the current higher order material. In Cartesian components, this reads

$$
\begin{aligned}
\int_{V}\left(\sigma_{i j} \delta \varepsilon_{i j}+\left(q_{i j}-s_{i j}\right) \delta \varepsilon_{i j}^{p}\right. & \left.+\tau_{i j k} \delta \varepsilon_{i j, k}^{p}\right) \mathrm{d} V \\
& =\int_{S}\left(T_{i} \delta u_{i}+M_{i j} \delta \varepsilon_{i j}^{p}\right) \mathrm{d} S .
\end{aligned}
$$

where $q_{i j}$ is the micro-stress tensor, $\sigma_{i j}$ is the Cauchy stress tensor, $s_{i j}=\sigma_{i j}-\delta_{i j} \sigma_{k k} / 3$ is the stress deviator and $\tau_{i j k}$ is the higher order stresses, work conjugate to the plastic strain gradients, $\varepsilon_{i j, k}^{p}$. Here, ()$_{, k}$ denotes the partial derivative with respect to the coordinate $x_{k}$. The right-hand side of Eqn. (1) includes both conventional tractions, $T_{i}=\sigma_{i j} n_{j}$, and higher order tractions, $M_{i j}=\tau_{i j k} n_{k}$, with $n_{k}$ denoting the outward normal to the surface $S$, which bounds the volume $V$.

The mechanisms associated with dislocation movement and/or storage of geometrically necessary dislocations (GND's) [7, 19, 22] have been incorporated into the current higher order theory by assuming the micro-stress, $q_{i j}$, and higher order stress, $\tau_{i j k}$, to have a dissipative part only, such that; $q_{i j}=q_{i j}^{D}$, and $\tau_{i j k}=\tau_{i j k}^{D}$ (all energetic contributions are omitted). These dissipative stress quantities read $[11,15]$

$$
q_{i j}^{D}=\frac{2}{3} \frac{\sigma_{C}\left[\dot{E}^{p}, E^{p}\right]}{\dot{E}^{p}} \dot{\varepsilon}_{i j}^{p}, \quad \tau_{i j k}^{D}=\frac{\sigma_{C}\left[\dot{E}^{p}, E^{p}\right]}{\dot{E}^{p}}\left(L_{D}\right)^{2} \dot{\varepsilon}_{i j, k}^{p}
$$

while the associated effective stress measure is

$$
\sigma_{C}=\sqrt{\frac{3}{2} q_{i j}^{D} q_{i j}^{D}+\left(L_{D}\right)^{-2} \tau_{i j k}^{D} \tau_{i j k}^{D}}
$$


Table 1. Material properties.

\begin{tabular}{lll}
\hline Parameter & Significance & Value \\
\hline$\sigma_{y} / E$ & Yield strain & $0.001-0.003$ \\
$v$ & Poisson's ratio & 0.3 \\
$N$ & Strain hardening exponent & $0.1-0.2$ \\
$m$ & Strain rate hardening exponent & 0.01 \\
$\dot{\varepsilon}_{0}$ & Reference strain rate & 0.002 \\
$L_{D} / H$ & Dissipative length parameter & $0.05-1.00$ \\
\hline
\end{tabular}

Assuming the form of the free energy to be

$$
\Psi=\frac{1}{2}\left(\varepsilon_{i j}-\varepsilon_{i j}^{p}\right) \mathcal{L}_{i j k l}\left(\varepsilon_{k l}-\varepsilon_{k l}^{p}\right)
$$

the conventional stresses are derived as; $\sigma_{i j}=\partial \Psi / \partial \varepsilon_{i j}^{e}=$ $\mathcal{L}_{i j k l}\left(\varepsilon_{k l}-\varepsilon_{k l}^{p}\right)$, where $\mathcal{L}_{i j k l}$ is the isotropic elastic stiffness tensor. Moreover, a power-law relation for the visco-plastic behaviour is assumed, so that

$$
\dot{E}^{p}=\dot{\varepsilon}_{0}\left(\frac{\sigma_{C}}{g\left(E^{p}\right)}\right)^{1 / m}, \text { with } g\left(E^{p}\right)=\sigma_{y}\left(1+\frac{E E^{p}}{\sigma_{y}}\right)^{N}
$$

where $N$ is the strain hardening exponent, $m$ is the strain rate hardening exponent, and $\dot{\varepsilon}_{0}$ is the reference strain rate. Thus, $\sigma_{C}\left[E^{p}, \dot{E}^{p}\right]=g\left(E^{p}\right)\left(\dot{E}^{p} / \dot{\varepsilon}_{0}\right)^{m}$.

In this model, the visco-plastic behaviour becomes significant for larger values of the strain rate hardening exponent, $m$, while the current constitutive material model approaches the response of a gradient enhanced J2-flow type material in the rate-independent limit $(m \rightarrow 0$, see e.g. $[15,23,24])$. Moreover, the response of the gradient enhanced model reduces to the prediction of its corresponding conventional version as the length parameter goes to zero $\left(L_{D}=0\right)$. The numerical framework is, however, unstable in the limit when $L_{D} \rightarrow 0$, and thus a conventional material model has been independently developed and coded for comparison. The material properties considered in the present study are summarized in Tab. 1.

\subsection{Steady-State Formulation}

The steady-state finite element (FE) formulation proposed by Dean and Hutchinson [21] is chosen over a classical transient Lagrangian modeling approach since it directly brings out the steady-state field that appears stationary relative to the rolls. Thus, convergence issues of any transient behaviour are avoided, making the steady-state formulation more precise and less demanding in terms of calculation time (see also [6] for a similar approach). Moreover, the modeling approach directly accommodates elastic-plastic unloading, and can be adapted to a wide range of constitutive models.
Dean and Hutchinson [21] originally define steady-state in the context of crack propagation as the condition at which the stress/strain field surrounding an advancing crack tip remains unchanged to an observer moving with the tip. A similar approach can be adopted for the rolling process, where stationarity of the stress/strain field must exist for a continuous feed of a homogeneous sheet. Thus, the stress/strain field remains unchanged to an observer at the rolls seeing the material pass by. Any time derived quantity, $\dot{f}$, in the constitutive model can thereby be related to the spatial derivative through the sheet velocity, $\dot{a}$, along the $x_{1}$-direction, according to $\dot{f}=-\dot{a} \frac{\partial f}{\partial x_{1}}$. An incremental quantity, at a given material point $\left(x_{1}^{*}, x_{2}^{*}\right)$, can thereby be evaluated by a streamline integration along the negative $x_{1}$-direction (see Fig. 1), which starts well in front of the active plastic zone (upstream, $\left.x_{1}=x_{1}^{0}>>0, x_{2}=x_{2}^{*}\right)$ and ends at the point of interest $\left(x_{1}=x_{1}^{*}, x_{2}=x_{2}^{*}\right)$. E.g. the plastic strains are determined as;

$$
\varepsilon_{i j}^{p}\left(x_{1}^{*}, x_{2}^{*}\right)=\int_{x_{1}^{0}}^{x_{1}^{*}} \frac{\partial \varepsilon_{i j}^{p}}{\partial x_{1}} d x_{1}, \quad \text { with } \quad \frac{\partial \varepsilon_{i j}^{p}}{\partial x_{1}}=-\frac{1}{\dot{a}} \dot{\varepsilon}_{i j}^{p}
$$

and $\dot{\varepsilon}_{i j}^{p}$ being the plastic strain rates. The spatial streamline integration is carried out using a standard forward Euler time integration, with the point of interest holding the history of all upstream material points.

In the chosen model formulation, the conventional principle of virtual work for quasi-static problems can be use to determine the displacement field, $u_{i}$ (which corresponds to Minimum Principle II in [15]),

$$
\int_{V} \mathcal{L}_{i j k l} \varepsilon_{k l} \delta \varepsilon_{i j} \mathrm{~d} V=\int_{S} T_{i} \delta u_{i} \mathrm{~d} S+\int_{V} \mathcal{L}_{i j k l} \varepsilon_{k l}^{p} \delta \varepsilon_{i j} \mathrm{~d} V
$$

whereas a corresponding minimum principle can be formulated for the plastic strain rate field (which corresponds to Minimum Principle I in [15]),

$$
\begin{aligned}
& \int_{V}\left(q_{i j}^{D} \delta \dot{\varepsilon}_{i j}^{p}+\tau_{i j k}^{D} \delta \dot{\varepsilon}_{i j, k}^{p}\right) \mathrm{d} V \\
&=\int_{V} s_{i j} \delta \dot{\varepsilon}_{i j}^{p} \mathrm{~d} V+\int_{S} M_{i j} \delta \dot{\varepsilon}_{i j}^{p} \mathrm{~d} S .
\end{aligned}
$$

The displacement field, and the related plastic strain rate field can thereby be iterated upon in a "staggered" approach.

The numerical implementation follows that of Niordson and Hutchinson [25]. Thus, based on the Minimum Principles in Eqns. (7)-(8), a standard finite element interpolation of the form

$$
\dot{u}_{i}=\sum_{n=1}^{8} N^{(n)} \dot{u}_{i}^{(n)} \quad \text { and } \quad \dot{\varepsilon}_{i j}^{p}=\sum_{n=1}^{4} N^{(n)} \dot{\varepsilon}_{i j}^{p(n)}
$$

can be introduced for the displacement increments and the plastic strain rate field, respectively. Here, 8-node isoparametric plane strain elements are used for the discretization 


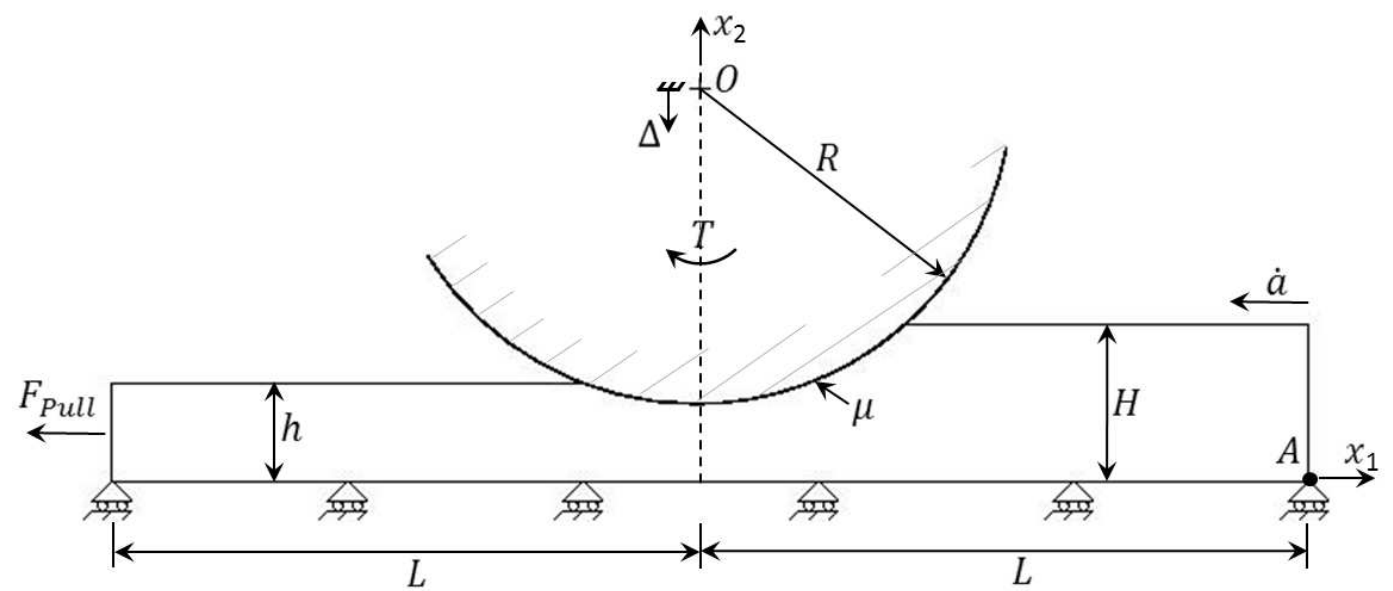

Fig. 1. Parametrization of the rolling process under steady-state conditions, with symmetry applied at $x_{2}=0$. Throughout, $\dot{a} /\left(\dot{\varepsilon}_{0} H\right)=50$, $L / H=10$, with the domain discretized by equal sized squared elements of side length; $L^{(e)} / H=20$, and unit thickness. Not shown is the width of the sheet in the out-of-plane direction, $b$.

of the displacement field, and corresponding 4-node elements are used for the plastic strain rate field. Both element types are integrated using Gauss quadrature, with $2 \times 2$ Gauss points. The nodal solution is iterated upon following a steady-state integration procedure similar to that in [21,26-28]. A detailed overview of the algorithm can be found in [29].

\section{Problem Formulation}

In steady-state plane strain rolling the translational displacement of the sheet is large, but the strains remain small for moderate thickness reductions. E.g. let the position of a material point at time $t=0$ be $\left(x_{1}, x_{2}\right)$ in the coordinate system defined in Fig. 1, and denote the position of this same material point at time $t$ by $\left(X_{1}, X_{2}\right)$. The displacements, $u_{i}\left(x_{1}, x_{2}\right)$, used in the formulation are defined by the relation of these two positions of the same material point:

$$
X_{1}=x_{1}-\dot{a} t+u_{1}, \quad X_{2}=x_{2}+u_{2}
$$

where $\dot{a}$ is the constant velocity in the negative $x_{1}$-direction of the undeformed sheet feeding into the rolls. The displacements, $u_{i}\left(x_{1}, x_{2}\right)$, are relative to a frame translating with the feed velocity of the sheet, so that the strains are: $\varepsilon_{i j}=\left(u_{i, j}+u_{j, i}\right) / 2$. Each iteration in the process to solve for the displacements, strains and plastic strains in the current model set-up involves two sequential steps: i) solving for the distribution of plastic strain rates, $\dot{\varepsilon}_{i j}^{p}$, using Minimum Principle I in Eqn. (8) and obtaining the plastic strains by integrating along the streamlines as in Eqn. (6); and ii) using Minimum Principle II in Eqn. (7) to obtain the displacements and strains.

The thickness of the undeformed sheet feeding into the rolls is $2 H$. The two circular cylindrical rolls of radius, $R$, are considered to be rigid and the downward displacement, $\Delta$, of the upper roll is the depth of the minimum point on the roll surface below $x_{2}=H$ (see Fig. 1). The lower roll is assumed to be displaced upward by the same amount, thus symmetry conditions are enforced along $x_{2}=0$ with $u_{2}\left(x_{1}, 0\right)=0$ and $\dot{\varepsilon}_{12}^{p}\left(x_{1}, 0\right)=0$, such that only the upper half of the sheet is meshed. The prescribed punch displacement is $\Delta / H$, which is the thickness reduction, $(H-h) / H$, plus a small elastic spring-back, c.f., Fig. 1. In addition to the material properties and the parameters controlling the rolling configuration $(R / H, \Delta / H)$, a dimensionless feed velocity of $\dot{a} /\left(H \dot{\varepsilon}_{0}\right)=50$ is also prescribed. For the small values of the strain rate hardening exponent, $m$, used to obtain the results in this study, there is virtually no dependence on the feed velocity, i.e. the rolling process is essentially modeled rate-independent (for a rate-dependent study see e.g. [30]).

As will become evident in the discussion to follow, some load cases require an added pull force, $F_{p u l l} / H b \sigma_{y}$, to ensure equilibrium. The pull force and the dimensionless torque applied to the roll, $T /\left(H b \sigma_{y} R\right)$, are evaluated using the tractions at the interface between the roll and the sheet, as-well as those at the end of the sheet (at $x_{1}=-L$ ). The model is formulated so that the prescribed pull force is overruled if it, combined with the roll torque, is insufficient to make the prescribed sheet reduction (the plate stops), or if it violates equilibrium (the plate accelerates). In both cases, the pull force is set to obey equilibrium in order to achieve the prescribed (constant) sheet velocity, $\dot{a}$. No force acts on the sheet in front of the roll. To enforce this condition, a displacement at the right end of the sheet, $u_{1}(L, 0)=\Delta_{A}$, is prescribed and adjusted each iteration such that the reaction force at this point is nearly zero (on the order of $10^{-6}$ of the pull force) in the converged solution. This constraint is enforced solely for numerical reasons and prevents free body motions.

To initiate the iterative steady-state procedure, the roll is first punched into the plate while restricting the movement of all nodes that interact with its surface, so that these can only slide along the circular path outlined by the rigid roll. For this, a linear constraint is readily derived, and enforced by a penalty approach. In this way a stress/strain field is created, where after the streamline integration of the constitutive equations can be carried out. In the subsequent iterations, 
the reaction forces on the roll are continuously checked, and nodes are left free to move in the case a negative pressure on the roll develops.

A "Stick-Slip" condition is employed at the contact interface so that the friction forces obey Coulomb friction, whenever slipping occurs, while the material is allowed to stick to the roll whereby the friction forces are set by static equilibrium. Thus, the maximum friction forces that can be transferred between the surfaces are; $F_{f, \max }=\mu F_{n}$, with $\mu$ being the friction coefficient. To ease the numerical analysis, all calculations are started by letting the algorithm converge without any sticking, whereafter this additional constraint is taken into account. Under full slipping (free sliding), the friction forces are introduced by first calculating the absolute sum of all nodal friction forces based on a kinematic Coulomb model $\left(F_{f}=\mu F_{n}\right)$, whereafter the direction of the individual nodal friction forces are determined from static equilibrium. Doing so, a discontinuous jump in the friction forces will occur at the neutral point. Once the algorithm has converged, the "Stick-Slip" condition is turned on, and sticking is indirectly accounted for by imposing the constraint that the straining in the rolling direction (along $x_{1}$ ), at the top surface, has to increase monotonically during contact. In case a decrease in straining occurs, the applied nodal friction force will be down-scaled to mimic static friction conditions (with $F_{f, \max }=\mu F_{n}$ ). The down-scaling and adjustment of the friction forces are made continuously during the iterative solution procedure, and allows for a "Stick" region to evolve (where the friction force gradually change direction, see e.g. Figs. 5-6).

\section{Results}

To demonstrate the behavior and capability of the steady-state model, a first comparison between the gradient enriched material model (described in Section 2.1), and the independently formulated conventional J2-flow model is laid out in Fig. 2 for a case with low friction $(\mu=0.005)$. The results in this figure are a subset of the full set of possible solutions associated with the prescribed punch displacement, $\Delta / H$. The solution in Fig. 2 is that corresponding to the minimum possible pull force for which a solution exists. Figure $2 \mathrm{a}$ is a plot of the dimensionless torque applied to the roll, and Fig. 2b gives the associated minimum dimensionless pull force. The gradient enhanced results were computed with a small material length parameter, $L_{D} / H=0.05$, such that gradient effects are also expected to be small. The close agreement with the prediction computed using a different code designed for conventional $\mathbf{J} 2$ plasticity theory is notable, giving confidence in the code for the strain gradient plasticity formulation. In Fig. 2 it is seen that the torque is positive and thus, at the minimum pull force, the roll torque and the pull force both do positive work on the sheet.

The full set of solutions for the same set of parameters as those in Fig. 2 is presented in Fig. 3. To understand the plots in Fig. 3, first note that the results for the minimum pull force constitute the lower curve for the pull force and the upper curve for the roll torque. The other limit, corre-

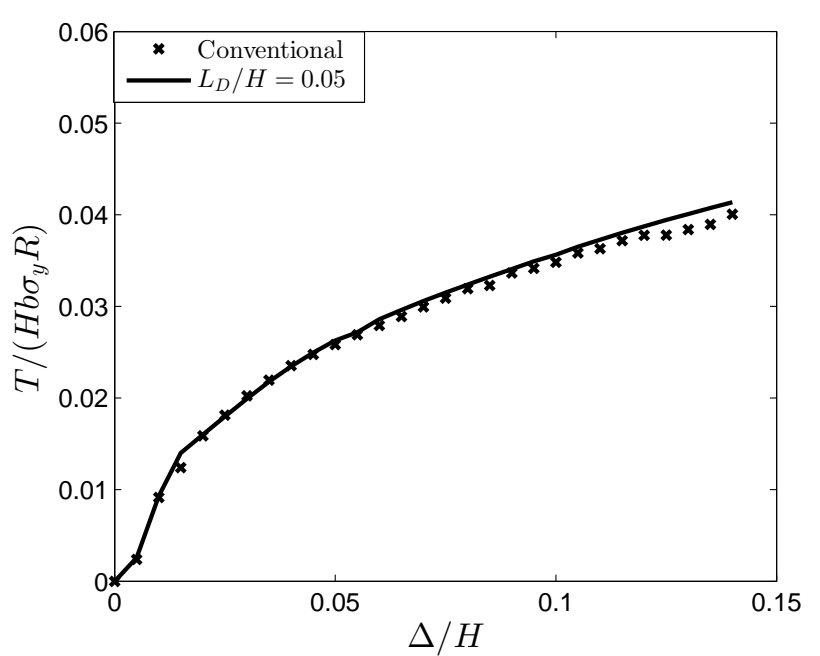

(a)

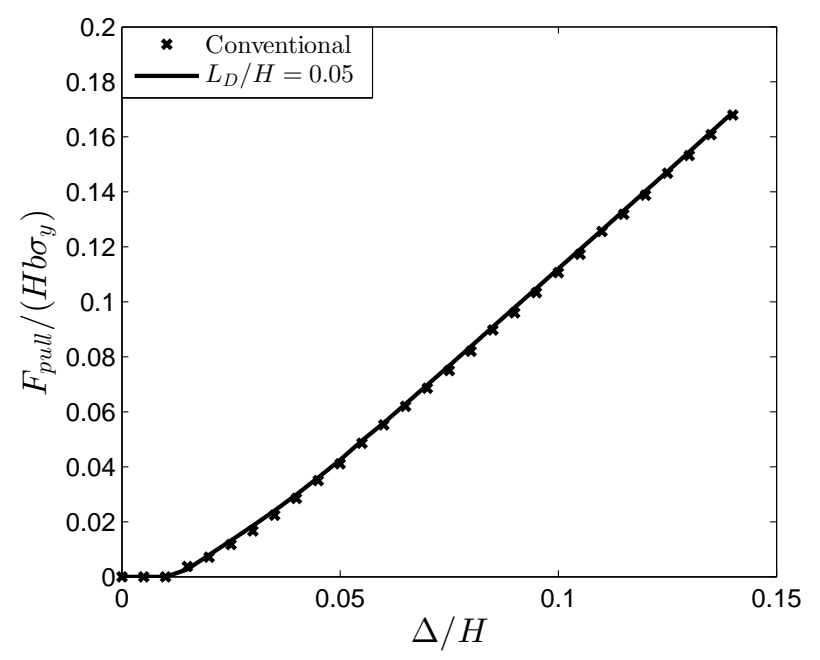

(b)

Fig. 2. Rolling at large scales $\left(L_{D} / H=0.05\right)$ with low friction $(\mu=$ 0.005 ), and minimum prescribed pull force. Here, showing a) applied torque, and b) applied pull force for various punch displacements, $\Delta / H\left(N=0.1, m=0.01, \sigma_{y} / E=0.003, R / H=100\right.$, and stick-slip condition active). The width of the out-of-plane direction is denoted $b$.

sponding to the maximum allowable pull force for any prescribed $\Delta / H$ is given by the upper curve for the pull force and the lower curve for the roll torque. Note that the roll torque corresponding to the maximum pull force is negative, resisting the motion of the sheet. The dimensionless punch force, being the required loading on the roll perpendicular to the sheet top surface, is plotted in Fig. $3 \mathrm{c}$ and is seen to be almost independent of the applied pull force. The dimensionless power expended by the combined pull force and the roll torque is plotted in Fig. $3 \mathrm{~d}$ with the upper curve corresponding to the maximum pull force, implying a negative roll torque $(T<0)$, whereas the lower curve corresponding to a positive roll torque $(T>0)$. The solutions corresponding to the minimum pull force, and thus minimum power require- 


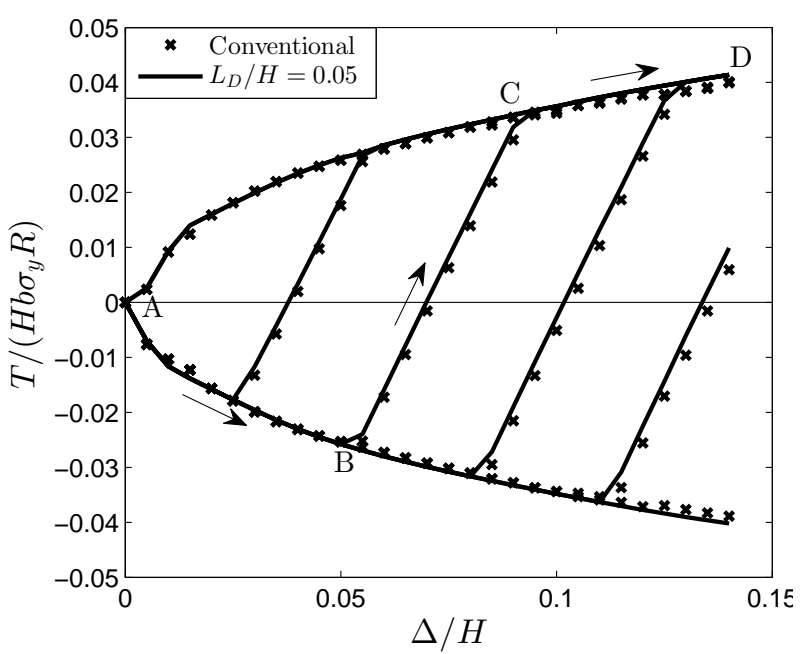

(a)

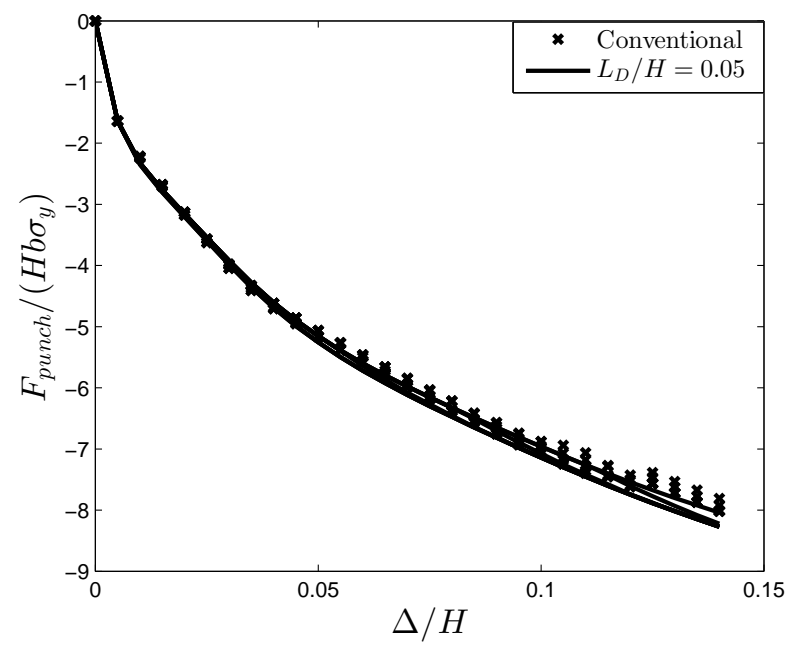

(c)

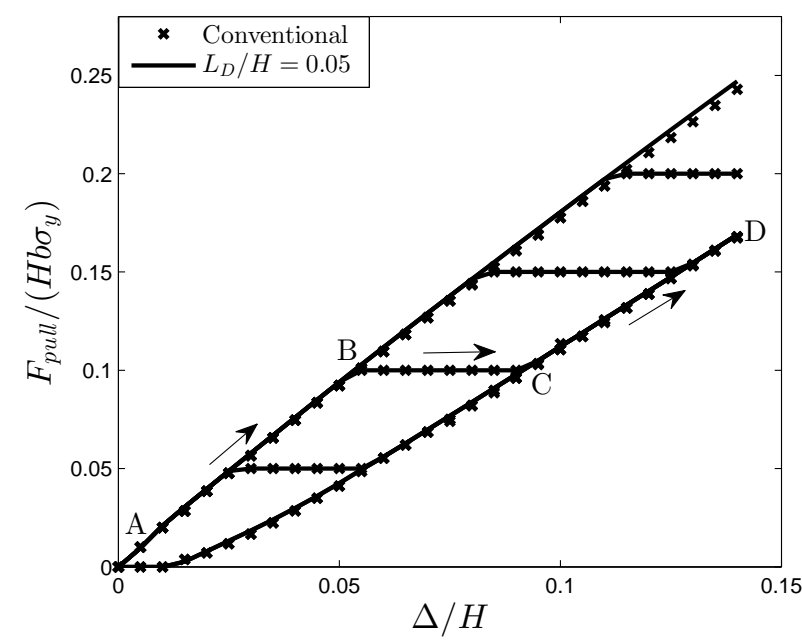

(b)

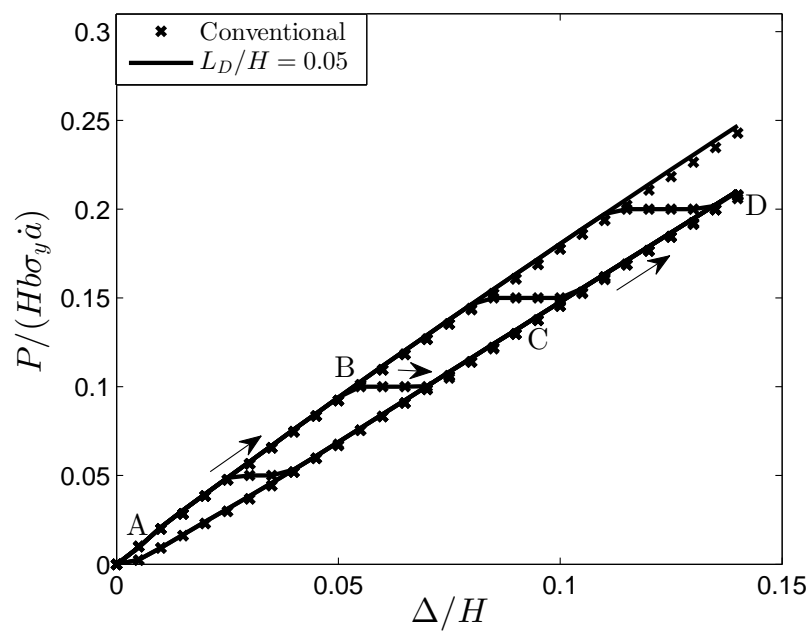

(d)

Fig. 3. Rolling at large scales $\left(L_{D} / H=0.05\right)$ with low friction $(\mu=0.005)$, and minimum prescribed pull force. Here, showing a) applied torque, b) applied pull force, c) applied punch force, and d) input power determined as $P=\left(F_{\text {pull }}+T / R\right) \dot{a}$ for $T>0$ or $P=F_{\text {pull }} \dot{a}$ for $T \leq 0$ for various punch displacements, $\Delta / H\left(N=0.1, m=0.01, \sigma_{y} / E=0.003, R / H=100\right.$, and stick-slip condition active). The width of the out-of-plane direction is denoted $b$.

ment, will have much of our attention.

For a prescribed punch displacement of $\Delta / H$, solutions exist for all pull forces lying between the lower and upper limits in Fig. 3. A selection of these intermediate solutions are plotted in each part of Fig. 3 associated with the curves connecting the minimum pull force limit to the maximum pull force limit, as will be discussed below in more detail.

Following the path "A-B-C-D" indicated in Fig. 3, for a prescribed pull force of $F_{\text {pull }}=H b \sigma_{y} / 10$, the model goes through three different stages as the punch displacement, $\Delta / H$, gradually increases; From " $\mathrm{A}$ " to "B", the prescribed pull force is too large for the combination of punch displacement and friction that has been specified, and thus the sheet would accelerate if the pull force were to be kept at its current level. This in spite the roll acts as to brake the sheet $(T<0)$. The prescribed pull force is therefore overruled to limit the power input (see Fig. 3b), and ensure equilibrium. At point
"B", the specified combination of punch displacement and friction gives rise to a braking torque coming from the roll that exactly matches the prescribed pull force, and equilibrium can be fulfilled without having to make additional adjustments. Seen form an energy point of view, this is the most inefficient way of producing the specified sheet reduction as the rolls counteracts the power input coming from the pull force (see Fig. 3d). From "B" to "C", the punch displacement has increased to a level where the roll torque is large enough to overcome the pull, and hence the prescribed pull force can be maintained (constant plateau in Fig. 3b). During this stage, the roll torque goes from braking the sheet $(T<0)$, through being allowed to spin freely ${ }^{1}(T=0)$, and to dragging the sheet between the rolls $(T>0)$. Thus, the

\footnotetext{
${ }^{1}$ This condition essentially corresponds to the so-called "English Wheel" used in metal forming, where the user exerts the pull force while the rolls spin freely.
} 


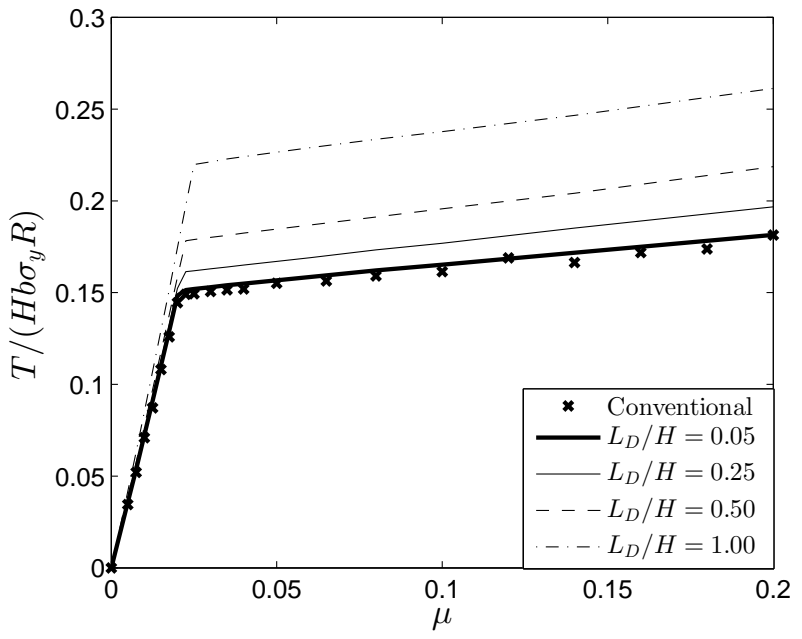

(a)

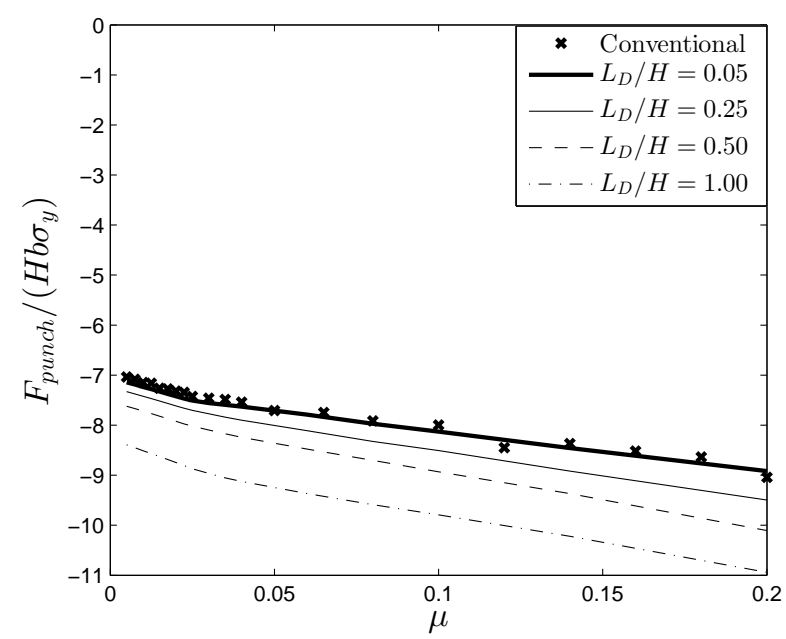

(c)

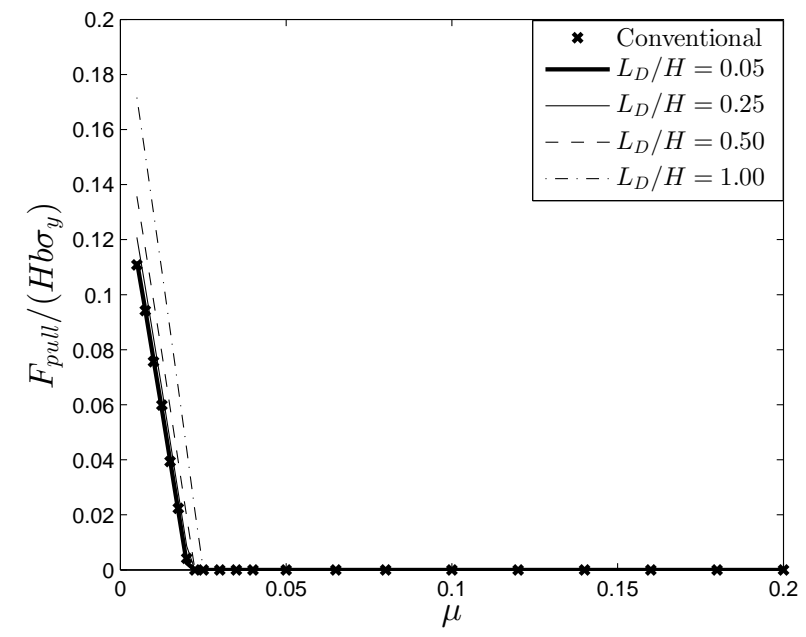

(b)

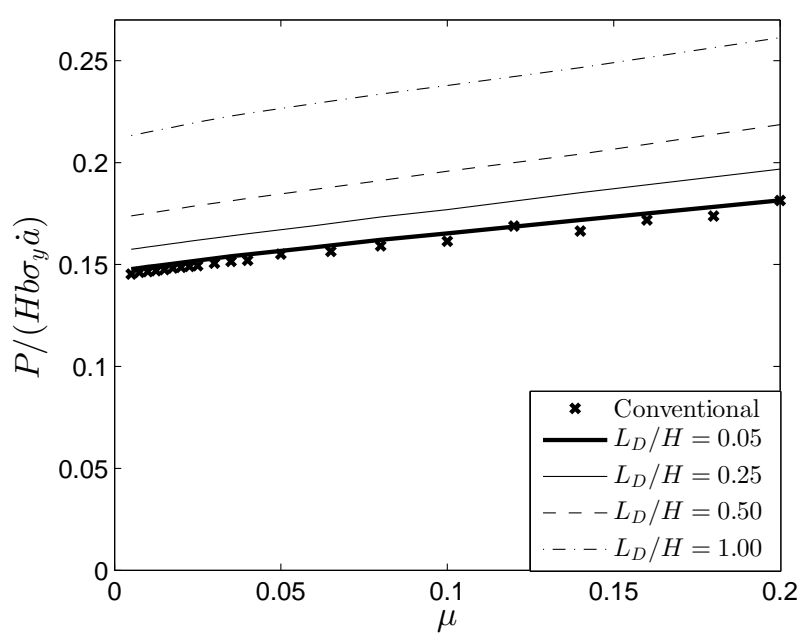

(d)

Fig. 4. Effect of friction on rolling at different scales with minimum prescribed pull force. Here, showing a) applied torque, b) applied pull force, c) applied punch force, and d) input power determined as $P=\left(F_{\text {pull }}+T / R\right) \dot{a}$ for $T>0$ or $P=F_{\text {pull }} \dot{a}$ for $T \leq 0$ for a fixed punch displacement of $\Delta / H=0.1$. Results are shown for various length scales, and with the stick-slip condition active $(N=0.1, m=0.01$, $\sigma_{y} / E=0.003$, and $R / H=100$ ). The width of the out-of-plane direction is denoted $b$.

model essentially shifts from the most inefficient branch and to the most efficient branch in the power input diagram in Fig. 3d. At point "C", the combined maximum torque transferred by the roll and the prescribed pull force act together, and match the forces needed to produce the specified sheet reduction - making this an energy efficient configuration (see Fig. 3d). However, any additional increase in punch displacement cannot be accommodated. This is also reflected in the final stage from " $C$ " to " $\mathrm{D}$ ", where an additional pull force is required to produced the specified sheet reduction. Due to the very low friction, the roll simply cannot deliver a sufficient torque (and power input), thus the pull force has to be increased to ensure a constant feed of the sheet.

It is worth noticing that the configuration of torque and pull belonging to a point in the upper half-space of Fig. 3a is tied to the lower branch of the power input (Fig. 3d) and hence to the most energy efficient way of producing a specific sheet reduction. This includes the upper branch for the torque (Fig. 3a), and the lower branch for the pull force (Fig. 3b). Moreover, solutions for constant sheet velocity only exist inside the branches brought out by Figs. 3a, b and $\mathrm{d}$, whereas the sheet either accelerates or decelerates for configurations above and below the branches, respectively.

The above example is an extreme case, and the friction level is typically much higher than $\mu=0.005$ in reality. During large scale cold rolling, Richelsen [5] estimates the friction coefficient to be on the order of $\mu \approx 0.1-0.2$, for a Wanheim-Bay type friction model $[31,32]$, and similar values are expected for the Coulomb friction model. For a sufficient friction level, the roll torque increases, and hence the pull force can be completely omitted in most cases. Model predictions for increasing friction is shown in Fig. 4 in order to illustrate this transition. Results are shown for a range of length parameters, and for a fixed punch displace- 


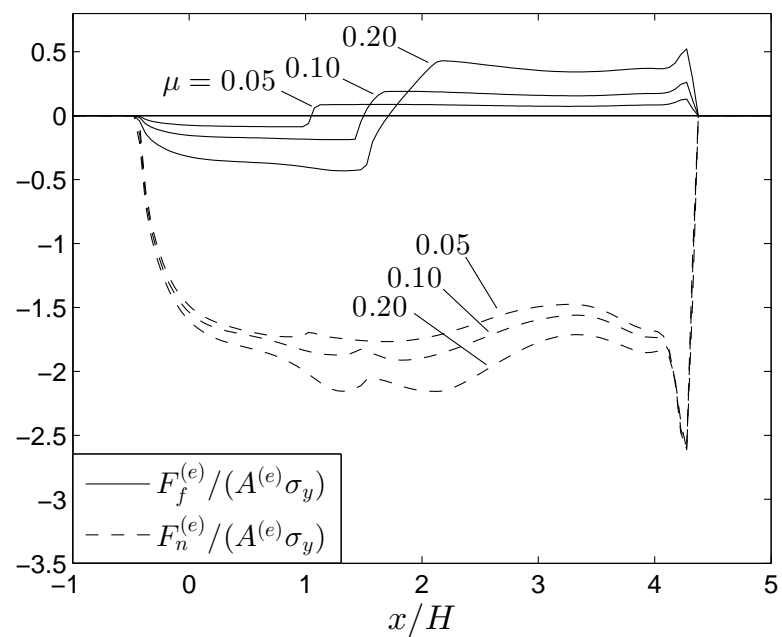

(a)

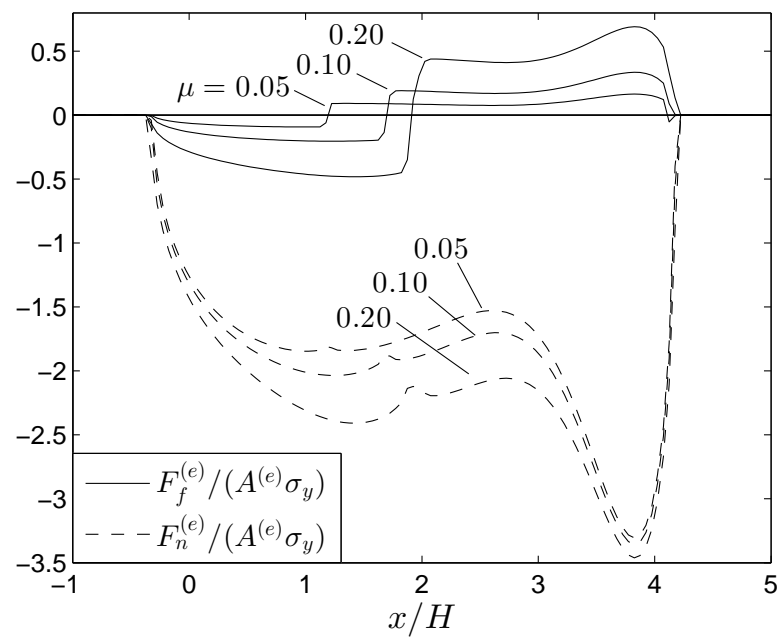

(b)

Fig. 5. Contact condition at the roll/sheet interface during rolling; a) at large scales $\left(L_{D} / H=0.05\right)$, and b) at small scales $\left(L_{D} / H=\right.$ 0.50 ). Here, showing the normalized friction forces (tangential traction) and normal forces (normal traction) for various friction levels $\left(N=0.1, m=0.01, \sigma_{y} / E=0.003, R / H=100, \Delta / H=0.1\right.$, and stick-slip condition active). Zero pull force is applied.

ment of $\Delta / H=0.1$. The smallest possible pull force is applied throughout these calculations. By increasing the friction from zero, it is seen that a pull force initially is needed to accommodate the specified sheet reduction since the roll cannot transfer sufficient torque to keep the rolling process going. However, the need for additional pull gradually diminishes as the level of friction increases (see Fig. 4b), and at $\mu \approx 0.02$, sufficient torque can be generated by the roll to maintain a constant velocity of the sheet. From here on, the pull force remains zero, while the roll torque increases (somewhat linearly) with the friction (compare Figs. 4a-b).

Figure 4 includes results for the effect of the length parameter, and clearly demonstrates the influence of size on the rolling process. As the length parameter increase (cor-

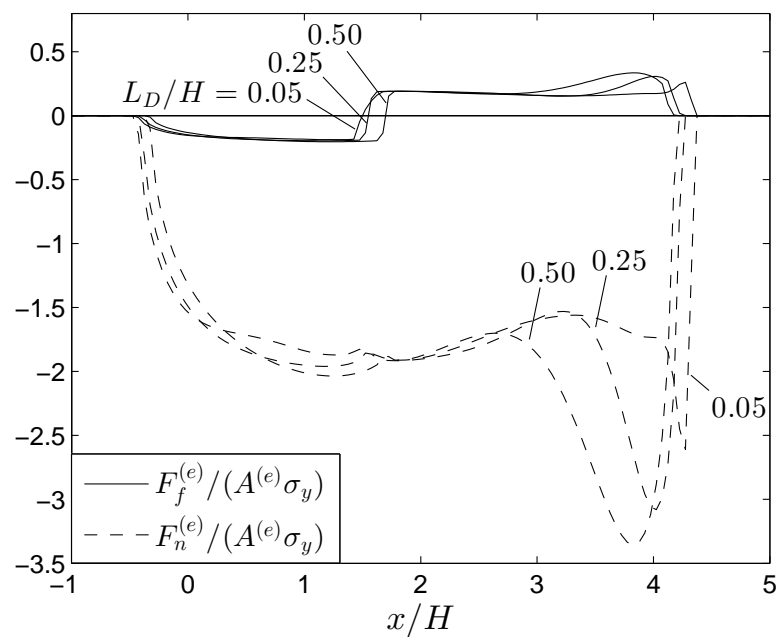

Fig. 6. Contact condition at the roll/sheet interface during rolling at different scales. Here, showing the normalized friction forces (tangential traction) and normal forces (normal traction) for various dissipative length parameters $\left(N=0.1, m=0.01, \mu=0.1, \sigma_{y} / E=\right.$ $0.003, R / H=100, \Delta / H=0.1$, and stick-slip condition active). Zero pull force is applied.

responding to rolling thinner sheets), the relative forces increase, and so does the stresses involved in the deformation process. This essentially means that the required power input increases relatively at small scale, which is also evident from Fig. 4d. This has to do with the increased yield resistance and hardening associated with GND's storage and movement in the lattice, and that the development of large strain gradients require additional energy. From Fig. $4 \mathrm{~b}$ it is noticed that the elevated forces at small scale leads to the requirement of higher friction before the pull force can be omitted, however in most practical settings this difference will be too small to be detected.

The contact conditions, at the interface between the rolls and sheet, is illustrated in Figs. 5-6 for various levels of friction and length parameters. The figures show the normalized contact forces (the traction) as function of position, with $F_{f}^{(e)}$ being the friction force, $F_{n}^{(e)}$ being the normal force, $A^{(e)}$ the element area, and $\sigma_{y}$ the yield stress ${ }^{2}$. Figure 5 compares rolling at a large scale $\left(L_{D} / H=0.05\right.$, see Fig. 5a) to rolling at a small scale $\left(L_{D} / H=0.5\right.$, see Fig. $\left.5 b\right)$ for different friction coefficients. Taking as off-set the large scale rolling, the predicted contact conditions compare well to previously published results (see e.g. [5]), both with respect to the level and distribution of the surface traction. In particularly, it is noticed that the steep peak in the normal force at first contact is captured, and so is the secondary, much smoother, peak near the stick region, where also the neutral point exists (and where the friction forces change direction). Moreover, a similar distribution for the absolute values of the friction forces

\footnotetext{
${ }^{2}$ The chosen configuration of the figures yield the cleanest representation of results, and a comparison with the stress level close to the top surface of the sheet shows good agreement.
} 

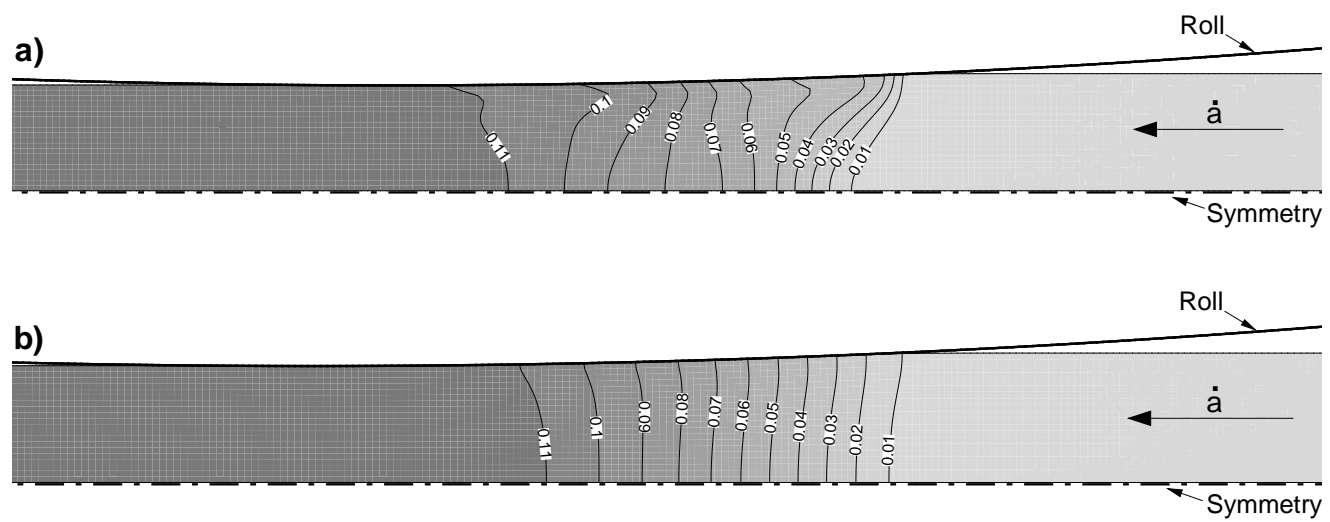

Fig. 7. Curves of constant effective gradient enhanced plastic strain, $E^{p}$, for a fixed punch displacement of $\Delta / H=0.1$, and zero pull force. Here, comparing two levels of the dissipative length parameter a) $L_{D} / H=0.05$, and b) $L_{D} / H=0.50(N=0.1, m=0.01$, $\sigma_{y} / E=0.003, R / H=100, \mu=0.1$, and stick-slip condition active).

is predicted, since; $F_{f}=\mu F_{n}$ during slipping, whereas their gradual shift in direction within the sticking region demonstrates a close match with the more computational demanding model by Richelsen [5]. Bear in mind that the current steady-state model provides the solution without having to deal with the transient behavior.

The distribution of tractions found under small scale rolling displays close similarities to those at larger scales (compare Fig. 5a-b). It is, however, noticed that the sticking region becomes comparably more narrow for rolling at small scale, and thus the change in direction for the friction forces is more abrupt. Moreover, it is clear that the contact forces evolving at small scale are relative higher, and a somewhat smoother peak is observed at the point of first contact. This is due to the increased hardening associated with the development of strain gradients, and thus the strain gradient effect tends to smear out the plastic strain field. The gradual change in the contact conditions when going from large to small scale is evident from Fig. 6. Results are, here, shown for a specified friction coefficient of $\mu=0.1$ and a fixed punch displacement of $\Delta / H=0.1$. The steep peak occurring at large scale $\left(L_{D} / H=0.05\right)$ gradually smoothens and increases as the length parameter increases (corresponding to rolling thinner sheets). This smoothening effect can also be seen directly from the plastic strain field. Figures $7 \mathrm{a}-$ b show the gradient enhanced effective plastic strain for the rolling process at large and small scale, respectively. Where the large scale rolling displays close lying contours near the first point of contact (hence large gradients), much more uniformly spaced strain contours are capture for rolling at small scale.

From geometrical arguments one can realize that the strain gradients become larger near the point of first contact in case the roll size decreases (keeping all other parameters fixed). This simply has to do with the sheet reduction taking place over a shorter distance. Thus, the size of the roll becomes important when accounting for length scale effects as large strain gradients significantly influence the material response. This is also clear from Figs. 8-9, where rolling at different scales and different roll sizes is demonstrated. Figure 8 shows the roll torque vs. the punch displacement for three different roll radii $(R / H \in[50,100,200])$ and two different scales. At large scale $\left(L_{D} / H=0.05\right)$, the model response is nearly independent of the size of the roll, and the prediction based on the gradient enriched model falls on top of that of the conventional model (employing a J2-flow material). On the contrary, the roll size does influence the response at the small scale $\left(L_{D} / H=0.5\right)$, and an relative increase in the required torque is observed when the roll radius becomes smaller (zero pull force is applied throughout). The results in Fig. 9 shows a similar trend. However, here showing the roll torque vs. the roll size for a fixed punch displacement of $\Delta / H=0.1$. As the roll size decreases the torque increases, especially at the very small scale $\left(L_{D} / H=1\right)$, whereas the response is nearly constant in the conventional limit. The associated punch forces are displayed in Fig. 9b.

The influence of conventional strain hardening is brought out in Fig. 10. Here, showing the torque transferred to the sheet, as-well as the punch force, for various length parameters, and two levels of strain hardening. Obviously, an increase in strain hardening leads to an increase in the forces involved in the rolling process. It is, however, seen that similar trends for the length scale parameter are obtained for both strain hardening values considered. Moreover, coinciding results with the corresponding J2-flow model are found in the conventional limit $\left(L_{D} / H \rightarrow 0\right)$.

A similar study has been carried out for the effect of the yield strain (/stress), and the results are collected in Appendix A.

\section{Conclusion}

Rolling at small scale is subject to the well-known size effects owing to strain gradient hardening as the deformation that takes place is non-homogeneous. Thus, a non-local material model has been adopted to accurately predict the ma- 


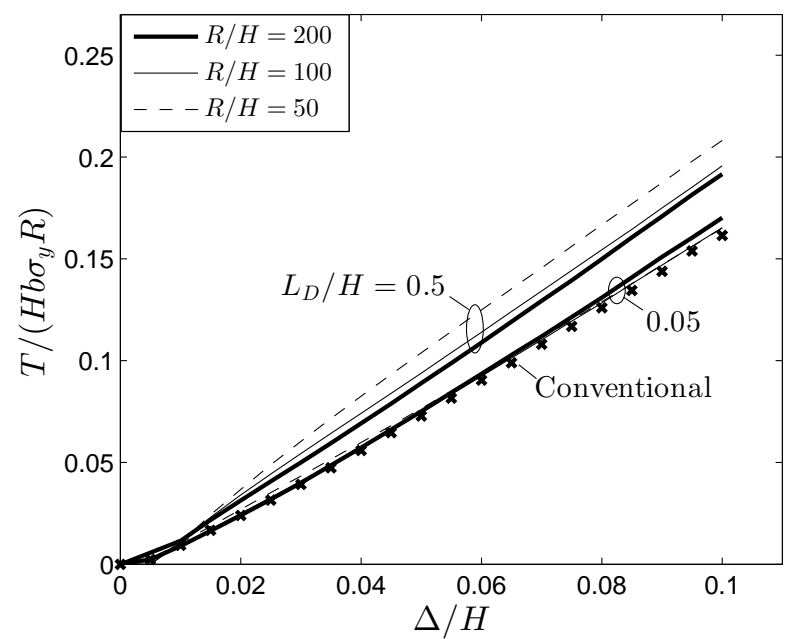

Fig. 8. Effect of roll size for various punch displacements, $\Delta / H$. Here, showing the applied torque with zero pull force prescribed. Results are shown for various dissipative length parameters, and with the stick-slip condition active $\left(N=0.1, m=0.01, \sigma_{y}=0.003\right.$, and $\mu=0.1$. Zero pull force is applied. The width of the out-ofplane direction is denoted $b$.

terial response over a range of length scales in order to bring out size effects. For a comprehensive study, the gradient enhanced elastic-viscoplastic material model by Fleck and Willis [15] has been employed, together with a steady-state technique, suitable for history dependent materials within the framework of finite element analysis. The developed modeling framework allows for an accurate representation of the material response over multiple length scales, including the prediction of elastic-plastic unloading and thus residual stresses and strains. The key findings of the study are;

i) Rolling at small scale require a relative larger power input, as additional energy is needed to develop strain gradients (see e.g. Figs. 3a and 4). Thus, for a given punch displacement, $\Delta / H$, a higher torque has to be generated at small scale to deform the sheet.

ii) The importance of sufficient friction, and the level required to reach a given sheet reduction has been demonstrated (see Fig. 4). As a specific case, the level of friction required to reach a sheet reduction of $\sim 10 \%$, without having to include additional pull to the sheet, is estimate to be $\mu \approx 0.02$, (for a strain hardening of $N=0.1$ ). This value, however, slightly increases as strain gradient becomes important.

iii) Down-scaling the rolling process influences the contact conditions. By adding strain gradient hardening a smoothed distribution of the interface traction is obtained, e.g. the peak at first contact becomes less sharp. Moreover, the level of both the normal and tangential (friction) traction increase at small scale - which relates directly to the relative larger forces involved (see Figs. 5-6).

iv) The roll size (i.e. the radius) becomes somewhat more important at small scales, and a detailed analysis shows strain gradient hardening to increase as the role size de-

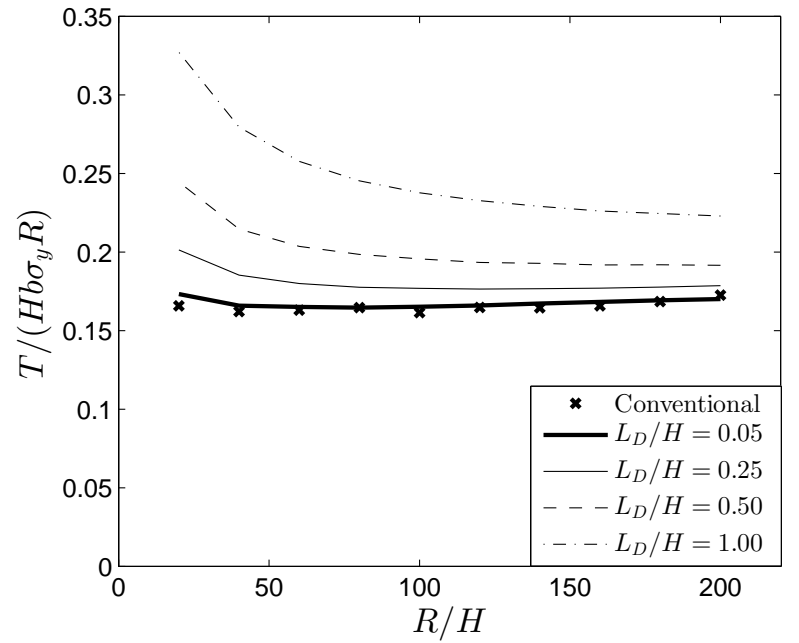

(a)

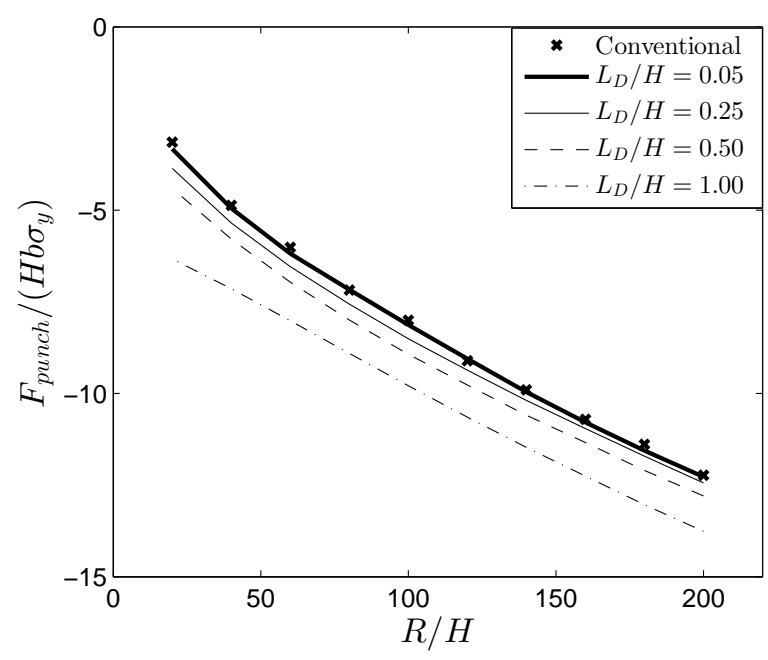

(b)

Fig. 9. Effect of roll size for a fixed punch displacement of $\Delta / H=$ 0.1 , showing a) applied torque, and b) applied punch force. Results are shown for various dissipative length parameters, and with the stick-slip condition active $\left(N=0.1, m=0.01, \sigma_{y} / E=0.003\right.$, and $\mu=0.1$ ). Zero pull force is applied. The width of the out-of-plane direction is denoted $b$.

creases (see Figs. 8-9). On the contrary, a negligible influence of the roll size is found for rolling at larger scale.

In summary, the present study has found that gradient effects begin to become noticeable when $L_{D} / H$ becomes roughly 0.25 . For many metal alloys, experimentally measured values of $L_{D}$ usually fall in the range from 1 to $5 \mu \mathrm{m}$. For metals with $L_{D}=5 \mu \mathrm{m}$, this implies that gradient effect should come into play when the sheet thickness $(2 H)$ is roughly $10 \mu \mathrm{m}$. It is also worth noting that the gradient effects lead to gradual modification of the predictions of conventional plasticity and at $L_{D} / H=1$, the modifications are significant but not dominating. 


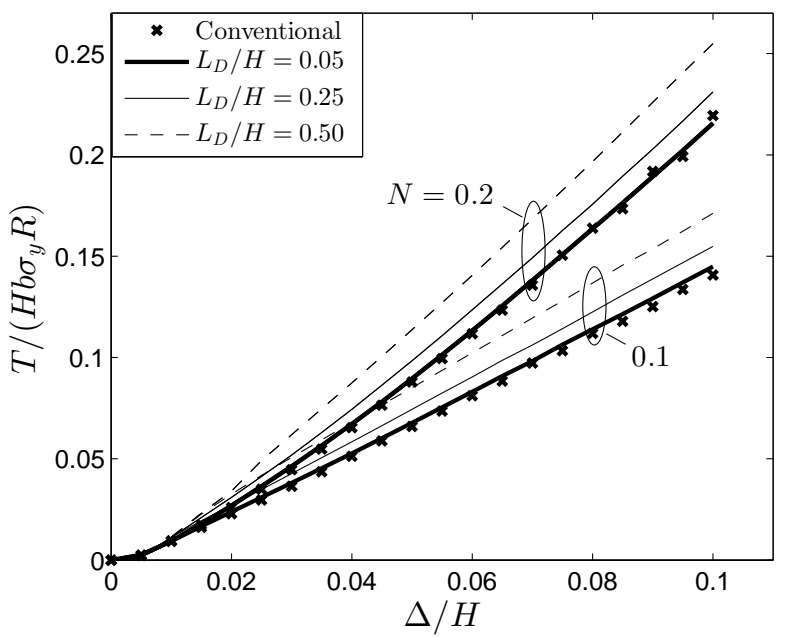

(a)

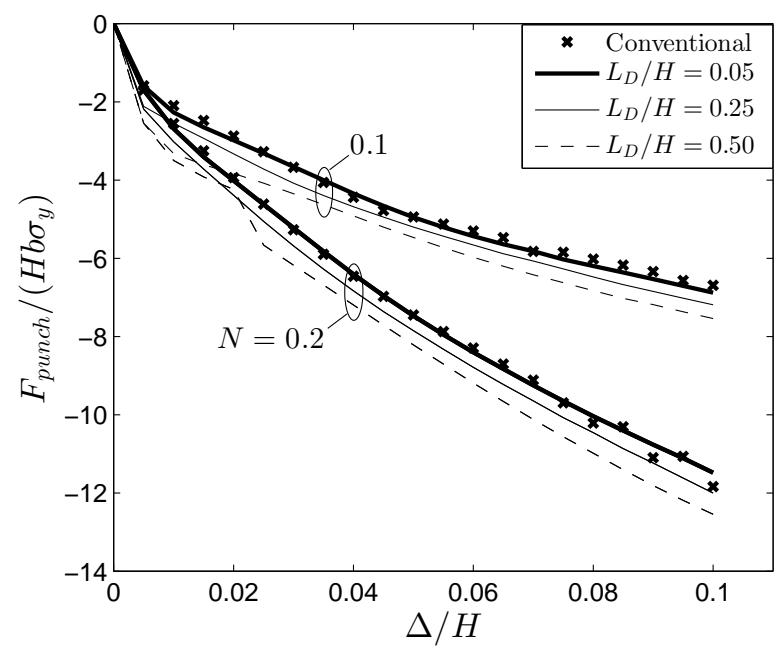

(b)

Fig. 10. Rolling at different scales with zero pull force. Here, showing a) applied torque, and b) applied punch force for various punch displacements, $\Delta / H$. Results are shown for various dissipative length parameters, and for two levels strain hardening $(N=[0.1,0.2])$ with the stick-slip condition active $(m=0.01$, $\sigma_{y} / E=0.003, R / H=100$, and $\mu=0.1$ ). The width of the outof-plane direction is denoted $b$.

\section{Achnowledgements}

The work is financially supported by The Danish Council for Independent Research under the research career programme Sapere Aude in the project "Higher Order Theories in Solid Mechanics".

\section{References}

[1] Montmitonnet, P., 2006. "Hot and cold strip rolling processes". Comp. Meth. in App. Mech. Eng., 193, pp. 6604-6625.

[2] Lu, B., and Meng, W., 2014. "Roll molding of microchannel arrays on $\mathrm{Al}$ and $\mathrm{Cu}$ sheet metals: a method for high-throughput manufacturing". ASME J. Micro Nano Manuf., 2(1), pp. 011007/1-9.

[3] Richelsen, A.-B., 1991. "Viscoplastic analysis of plane-strain rolling using different friction models". Int. J. Mech. Sci., 9, pp. 761-774.

[4] Richelsen, A.-B., 1994. "Interface element modelling of friction in rolling". J. Mater. Processing Technology, 42, pp. 209-216.

[5] Richelsen, A.-B., 1996. "Comparison of a numerical analysis of rolling with experimental data". J. Mater. Processing Technology, 57, pp. 70-78.

[6] Hacquin, A., Montmitonnet, P., and Guillerault, J.-P., 1996. "A steady state thermo-elastoviscoplastic finite element model of rolling with coupled thermoelastic roll deformation". J. Mater. Process. Techn., 60, pp. 109-116.

[7] Ashby, M., 1970. "The deformation of plastically nonhomogeneous alloys". Philos. Mag., 21, pp. 399-424.

[8] Aifantis, E., 1984. "On the Microstructural Origin of Certain Inelastic Models”. J. Eng. Mater. Techn., 106, pp. 326-330.

[9] Fleck, N., and Hutchinson, J., 1997. "Strain gradient plasticity". Adv. Appl. Mech., 33, pp. 295-361.

[10] Fleck, N., and Hutchinson, J., 2001. "A reformulation of strain gradient plasticity”. J. Mech. Phys. Solids, 49, pp. 2245-2271.

[11] Gudmundson, P., 2004. "A unified treatment of strain gradient plasticity”. J. Mech. Phys. Solids, 52, pp. 1379-1506.

[12] Gurtin, M., and Anand, L., 2005. "A theory of straingradient plasticity for isotropic, plastically irrotational materials. Part I: Small deformations". J. Mech. Phys. Solids, 53, pp. 1624-1649.

[13] Lele, S., and Anand, L., 2009. "A large-deformation strain-gradient theory for isotropic viscoplastic materials". Int. J. Plast., 25, pp. 420-453.

[14] Fleck, N., and Willis, J., 2009. "A mathematical basis for strain-gradient plasticity theory. Part I: Scalar plastic multiplier". J. Mech. Phys. Solids, 57, pp. 161-177.

[15] Fleck, N., and Willis, J., 2009. "A mathematical basis for strain-gradient plasticity theory. Part II: Tensorial plastic multiplier". J. Mech. Phys. Solids, 57, pp. 10451057.

[16] Hutchinson, J., 2012. "Generalized J2 flow theory: Fundamental issues in strain gradient plasticity". Acta Mech. Sinica, 28, pp. 1078-1086.

[17] Gao, H., Huang, Y., Nix, W., and Hutchinson, J., 1999. "Mechanism-based strain gradient plasticity I: analysis". J. Mech. Phys. Solids, 47, pp. 1239-1263.

[18] Huang, Y., Gao, H., Nix, W., and Hutchinson, J., 1999. "Mechanism-based strain gradient plasticity II: analysis". J. Mech. Phys. Solids, 48, pp. 99-128.

[19] Gurtin, M., 2002. "A gradient theory of single-crystal viscoplasticity that accounts for geometrically necessary dislocations". J. Mech. Phys. Solids, 50, pp. 5-32.

[20] Qiu, X., Huang, Y., Wei, Y., Gao, H., and Hwang, K., 2003. "The flow theory of mechanism-based strain gradient plasticity". Mech. Mater., 35, pp. 245-258. 
[21] Dean, R., and Hutchinson, J., 1980. "Quasi-Static Steady Crack Growth in Small-Scale Yielding". Fracture Mechanics: Twelfth Conference, ASTM STP700, American Society for Testing and Materials, pp. 383405.

[22] Ohno, N., and Okumara, D., 2007. "Higher-order stress and grain size effects due to self-energy of geometrically necessary dislocations". J. Mech. Phys. Solids, 55, pp. 1879-1898.

[23] Nielsen, K., and Niordson, C., 2013. "A 2D finite element implementation of the Fleck-Willis straingradient flow theory". Europ. J. Mech. A/Solids, 41, pp. 134-142.

[24] Nielsen, K., and Niordson, C., 2014. "A numerical basis for strain-gradient plasticity theory: rateindependent and rate-dependent formulations". $J$. Mech. Phys. Solids, 63, pp. 113-127.

[25] Niordson, C., and Hutchinson, J., 2011. "Basic strain gradient plasticity theories with application to constrained film deformation”. J. Mech. Mater. Struct., 6, pp. 395-416.

[26] Niordson, C., 2001. "Analysis of steady-state ductile crack growth along a laser weld". Int. J. Frac., 111, pp. 53-69.

[27] Wei, Y., and Hutchinson, J., 1997. "Steady-State crack growth and work of fracture for solids characterized by strain gradient plasticity". J. Mech. Phys. Solids, 45, pp. 1253-1273.

[28] Nielsen, K., and Niordson, C., 2012. "Rate sensitivity of mixed mode interface toughness of dissimilar metallic materials.”. Int. J. Solids. Struct., 49, pp. 576-583.

[29] Nielsen, K., Niordson, C., and Hutchinson, J., 2012. "Strain gradient effects on steady state crack growth in rate-sensitive materials". Eng. Frac. Mech., 96, pp. 6171.

[30] Nielsen, K., 2015. "Rolling induced size effects in elastic-viscoplastic sheet metals". Euro. J. Mech. A/Solids, 53, pp. 259-267.

[31] Bay, N., and Wanheim, T., 1976. "Real area of contact and friction stress at high pressure sliding contact". Wear, 38, pp. 201-209.

[32] Wanheim, T., and Bay, N., 1978. "A model for friction in metal forming processes". Ann. CIRP, 27, pp. 189194.

\section{Appendix A: Effect of Yield Strain at Different Scales}

The flat rolling process is adopted to a wide range of materials, and thus it is important to analyze the influence of the material parameter specifying first yield. Below is included a collection of results for the effect of the yield strain. The results are obtained by keeping Young's modulus fixed and altering the yield stress. By adopting the normalization used in the remaining figures, only a fairly limited influence of the yield strain is observed, and the effect is nearly identical at all scales (see Fig. 11).

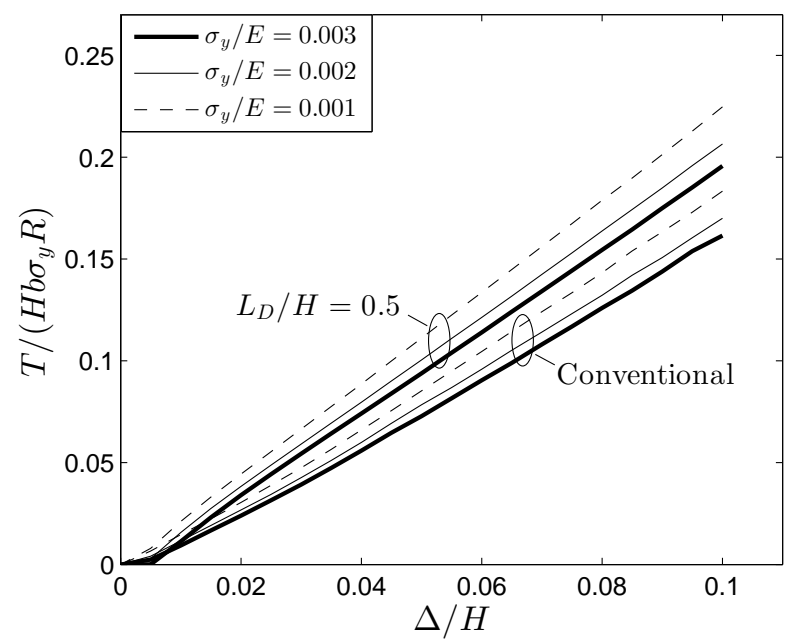

(a)

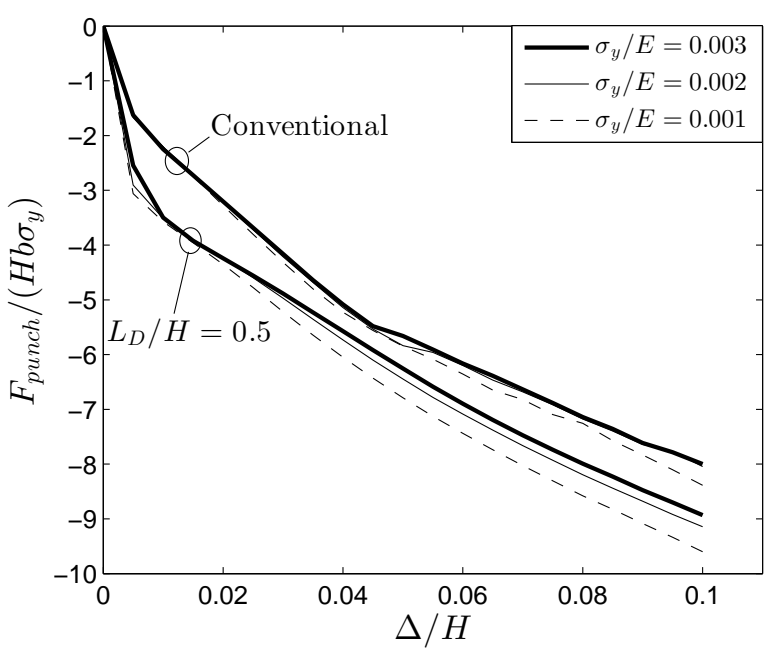

(b)

Fig. 11. Rolling at different scales with zero pull force. Here, showing a) applied torque, and b) applied punch force for various punch displacements, $\Delta / H$. Results are shown for three levels of initial yield strain $\left(\sigma_{y} / E=[0.001,0.002,0.003]\right)$, with the stick-slip condition active $(N=0.1, m=0.01, \mu=0.1$, and $R / H=100)$. The width of the out-of-plane direction is denoted $b$. 